\title{
The shape of 4(S)- and 4(R)-hydroxyproline in gas phase
}

\section{Tables of Supplementary Material}

\author{
Alberto Lesarri, Emilio J. Cocinero, Juan C. López and José L. Alonso
}

Grupo de Espectroscopía Molecular (GEM), Departamento de Química Física y Química Inorgánica, Facultad de Ciencias, Universidad de Valladolid, 47005 Valladolid, Spain.

7 Tables

10 Pages

Corresponding author:

José L. Alonso

Grupo de Espectroscopía Molecular,

Departamento de Química Física y Química Inorgánica, Facultad de Ciencias,

Universidad de Valladolid, 47005 Valladolid, Spain

Tel.: +34-983-423204 / Fax: +34-983-423204

E-mail: jlalonso@gf.uva.es 
Table S1. Observed frequencies and residuals $(\mathrm{MHz})$ for the parent species of conformer $1 \mathrm{a}_{1}$ of 4(S)-hydroxyproline.

\begin{tabular}{|c|c|c|c|c|c|c|c|c|c|c|c|c|c|c|c|c|c|c|c|}
\hline \multirow{2}{*}{$\begin{array}{l}\text { J' } \\
2\end{array}$} & \multicolumn{2}{|c|}{$K_{-1}^{\prime} K^{\prime}+1$} & \multirow{2}{*}{$\begin{array}{l}\text { J" } \\
1\end{array}$} & \multicolumn{2}{|c|}{$K^{\prime \prime} K^{\prime \prime+1}$} & \multirow[t]{2}{*}{$F^{\prime}$} & \multirow{2}{*}{$\frac{F^{\prime \prime}}{2}$} & \multirow{2}{*}{$\begin{array}{c}\text { obs. }^{a} \\
6868.900\end{array}$} & \multirow{2}{*}{$\begin{array}{l}\text { o.-c. }{ }^{b} \\
0.000\end{array}$} & \multirow{2}{*}{$\begin{array}{l}\text { J' } \\
3\end{array}$} & \multicolumn{2}{|c|}{$\mathrm{K}_{-1}^{\prime} \mathrm{K}^{\prime}+1$} & \multicolumn{3}{|c|}{ J" K"-1 K"+1 } & \multicolumn{2}{|c|}{$F^{\prime} F^{\prime \prime}$} & \multirow{2}{*}{$\begin{array}{c}\text { obs. } \\
8272.289\end{array}$} & \multirow{2}{*}{$\begin{array}{c}\text { o.-c. } \\
0.000\end{array}$} \\
\hline & 1 & 1 & & 0 & 1 & & & & & & 2 & 2 & 2 & 2 & 1 & 4 & 3 & & \\
\hline \multirow[t]{3}{*}{2} & 2 & 0 & 1 & 1 & 0 & 3 & 2 & 8999.458 & 0.004 & & & & & & & 3 & 2 & 8272.635 & 0.000 \\
\hline & & & & & & 2 & 1 & 8999.858 & -0.001 & & & & & & & 2 & 1 & 8272.096 & -0.001 \\
\hline & & & & & & 1 & 0 & 8998.450 & -0.003 & 4 & 0 & 4 & 3 & 0 & 3 & 5 & 4 & 10945.318 & 0.000 \\
\hline \multirow[t]{3}{*}{2} & 2 & 0 & 1 & 1 & 1 & 3 & 2 & 9116.788 & 0.003 & & & & & & & 4 & 3 & 10945.465 & 0.001 \\
\hline & & & & & & 2 & 1 & 9115.877 & 0.005 & & & & & & & 3 & 2 & 10945.334 & 0.004 \\
\hline & & & & & & 1 & 0 & 9117.764 & 0.001 & 4 & 1 & 3 & 3 & 1 & 2 & 5 & 4 & 11245.221 & -0.001 \\
\hline 2 & 2 & 1 & 1 & 1 & 0 & 3 & 2 & 8990.723 & -0.002 & & & & & & & 4 & 3 & 11245.289 & 0.001 \\
\hline & & & & & & 2 & 1 & 8991.198 & -0.002 & & & & & & & 3 & 2 & 11245.165 & 0.000 \\
\hline & & & & & & 1 & 0 & 8989.681 & -0.005 & 4 & 0 & 4 & 3 & 1 & 3 & 5 & 4 & 10080.616 & 0.001 \\
\hline 2 & 2 & 1 & 1 & 1 & 1 & 3 & 2 & 9108.055 & -0.001 & & & & & & & 4 & 3 & 10080.274 & 0.001 \\
\hline & & & & & & 2 & 1 & 9107.208 & -0.004 & & & & & & & 3 & 2 & 10080.796 & -0.002 \\
\hline & & & & & & 1 & 0 & 9108.996 & 0.001 & 4 & 1 & 3 & 3 & 0 & 3 & 5 & 4 & 12812.525 & 0.002 \\
\hline 3 & 0 & 3 & 2 & 0 & 2 & 4 & 3 & 8237.767 & 0.000 & & & & & & & 4 & 3 & 12811.614 & 0.000 \\
\hline & & & & & & 3 & 2 & 8237.886 & 0.000 & & & & & & & 3 & 2 & 12812.802 & -0.005 \\
\hline & & & & & & 2 & 1 & 8237.809 & -0.002 & 4 & 1 & 4 & 3 & 0 & 3 & 5 & 4 & 11643.938 & -0.001 \\
\hline & & & & & & 3 & 3 & 8238.299 & -0.003 & & & & & & & 4 & 3 & 11644.517 & -0.002 \\
\hline & & & & & & 2 & 2 & 8237.164 & -0.001 & & & & & & & 3 & 2 & 11643.842 & 0.002 \\
\hline 3 & 1 & 2 & 2 & 0 & 2 & 4 & 3 & 9805.068 & 0.001 & 4 & 1 & 4 & 3 & 1 & 3 & 5 & 4 & 10779.237 & 0.000 \\
\hline & & & & & & 3 & 2 & 9804.212 & 0.000 & & & & & & & 4 & 3 & 10779.327 & 0.000 \\
\hline & & & & & & 2 & 1 & 9805.448 & -0.004 & & & & & & & 3 & 2 & 10779.310 & 0.002 \\
\hline & & & & & & 3 & 3 & 9804.632 & 0.005 & 4 & 2 & 2 & 3 & 2 & 1 & 5 & 4 & 11107.320 & 0.002 \\
\hline & & & & & & 2 & 2 & 9804.807 & 0.001 & & & & & & & 4 & 3 & 11107.345 & 0.000 \\
\hline 3 & 1 & 2 & 2 & 1 & 1 & 4 & 3 & 8442.334 & 0.000 & 4 & 2 & 3 & 3 & 2 & 2 & 5 & 4 & 11022.932 & 0.000 \\
\hline & & & & & & 3 & 2 & 8442.428 & 0.000 & & & & & & & 4 & 3 & 11023.095 & -0.001 \\
\hline & & & & & & 2 & 1 & 8442.191 & -0.001 & & & & & & & 3 & 2 & 11022.891 & 0.001 \\
\hline & & & & & & 3 & 3 & 8441.894 & 0.000 & 4 & 3 & 1 & 3 & 3 & 0 & 5 & 4 & 11048.419 & -0.001 \\
\hline & & & & & & 2 & 2 & 8443.022 & 0.001 & & & & & & & 4 & 3 & 11048.703 & 0.002 \\
\hline 3 & 1 & 3 & 2 & 1 & 2 & 4 & 3 & 8091.380 & 0.001 & 4 & 3 & 2 & 3 & 3 & 1 & 5 & 4 & 11045.988 & 0.000 \\
\hline & & & & & & 3 & 2 & 8091.526 & 0.004 & & & & & & & 4 & 3 & 11046.277 & -0.001 \\
\hline & & & & & & 2 & 1 & 8091.507 & -0.003 & & & & & & & 3 & 2 & 11045.870 & 0.000 \\
\hline & & & & & & 3 & 3 & 8092.402 & -0.001 & 5 & 0 & 5 & 4 & 0 & 4 & 6 & 5 & 13625.672 & 0.001 \\
\hline & & & & & & 2 & 2 & 8090.142 & 0.000 & & & & & & & 5 & 4 & 13625.832 & 0.001 \\
\hline 3 & 1 & 3 & 2 & 0 & 2 & 4 & 3 & 9102.471 & 0.001 & & & & & & & 4 & 3 & 13625.672 & -0.003 \\
\hline & & & & & & 3 & 2 & 9103.079 & 0.001 & 5 & 1 & 4 & 4 & 1 & 3 & 6 & 5 & 14037.249 & 0.000 \\
\hline & & & & & & 2 & 1 & 9102.343 & 0.000 & & & & & & & 5 & 4 & 14037.319 & 0.002 \\
\hline 3 & 2 & 1 & 2 & 1 & 1 & 4 & 3 & 11674.297 & 0.002 & & & & & & & 4 & 3 & 14037.215 & 0.000 \\
\hline & & & & & & 3 & 2 & 11674.651 & -0.002 & 5 & 1 & 5 & 4 & 1 & 4 & 6 & 5 & 13460.234 & -0.001 \\
\hline & & & & & & 2 & 1 & 11674.060 & 0.001 & & & & & & & 5 & 4 & 13460.306 & -0.002 \\
\hline 3 & 2 & 1 & 2 & 2 & 0 & 4 & 3 & 8306.853 & -0.001 & & & & & & & 4 & 3 & 13460.280 & 0.000 \\
\hline & & & & & & 3 & 2 & 8307.092 & -0.001 & 5 & 2 & 3 & 4 & 2 & 2 & 6 & 5 & 13928.981 & 0.002 \\
\hline & & & & & & 2 & 1 & 8306.687 & 0.002 & & & & & & & 5 & 4 & 13928.929 & -0.002 \\
\hline 3 & 2 & 2 & 2 & 1 & 2 & 4 & 3 & 11982.646 & 0.003 & & & & & & & 4 & 3 & 13928.981 & 0.000 \\
\hline & & & & & & 3 & 2 & 11981.764 & 0.001 & 5 & 2 & 4 & 4 & 2 & 3 & 6 & 5 & 13767.727 & 0.002 \\
\hline & & & & & & 2 & 1 & 11983.130 & -0.002 & & & & & & & 5 & 4 & 13767.830 & 0.001 \\
\hline & & & & & & & & & & & & & & & & 4 & 3 & 13767.710 & -0.002 \\
\hline
\end{tabular}


Table S2. Observed frequencies and residuals $(\mathrm{MHz})$ for the monodeuterated isotopomers of conformer 1 $1 \mathrm{a}_{1}$ of 4(S)-hydroxyproline.

\begin{tabular}{|c|c|c|c|c|c|c|c|c|c|c|c|c|c|}
\hline & & & & & & \multirow[b]{2}{*}{$F^{\prime}$} & \multirow[b]{2}{*}{$F^{\prime \prime}$} & \multicolumn{2}{|c|}{ ND } & \multicolumn{2}{|c|}{ COOD } & \multicolumn{2}{|c|}{ OD } \\
\hline \multicolumn{3}{|c|}{$J^{\prime} K_{-1}^{\prime} K_{+1}^{\prime}$} & \multicolumn{3}{|c|}{$J^{\prime \prime} K^{\prime \prime}{ }_{-1} K^{\prime \prime}$} & & & \multirow{2}{*}{$\begin{array}{c}\text { obs. }^{\text {a }} \\
8156.717\end{array}$} & \multirow{2}{*}{$\begin{array}{l}\text { o.-c. }{ }^{b} \\
0.007\end{array}$} & \multirow{2}{*}{$\begin{array}{c}\text { obs. } \\
8184.943\end{array}$} & \multirow{2}{*}{$\begin{array}{l}\text { o.-c. } \\
-0.001\end{array}$} & \multirow{2}{*}{$\begin{array}{c}\text { obs. } \\
8174.485\end{array}$} & \multirow{2}{*}{$\begin{array}{c}\text { o.-c. } \\
0.002\end{array}$} \\
\hline 3 & 0 & 3 & 2 & 0 & 2 & 4 & 3 & & & & & & \\
\hline & & & & & & 3 & 2 & 8156.860 & 0.007 & 8185.058 & -0.001 & 8174.625 & 0.003 \\
\hline & & & & & & 2 & 1 & & & 8184.980 & -0.003 & 8174.510 & -0.010 \\
\hline \multirow[t]{3}{*}{3} & 1 & 2 & 2 & 1 & 1 & 4 & 3 & 8395.823 & 0.036 & 8395.568 & 0.001 & 8399.967 & -0.002 \\
\hline & & & & & & 3 & 2 & 8395.922 & 0.034 & 8395.653 & 0.000 & 8400.066 & 0.003 \\
\hline & & & & & & 2 & 1 & & & 8395.435 & 0.001 & 8399.817 & 0.000 \\
\hline \multirow[t]{3}{*}{3} & 1 & 3 & 2 & 1 & 2 & 4 & 3 & 7996.613 & 0.008 & 8036.694 & -0.001 & 8020.152 & 0.002 \\
\hline & & & & & & 3 & 2 & 7996.751 & -0.005 & 8036.829 & 0.002 & 8020.298 & 0.001 \\
\hline & & & & & & 2 & 1 & 7996.733 & -0.004 & 8036.810 & -0.004 & 8020.281 & -0.007 \\
\hline \multirow[t]{3}{*}{4} & 0 & 4 & 3 & 0 & 3 & 5 & 4 & 10824.577 & 0.003 & 10872.314 & -0.002 & 10852.999 & -0.004 \\
\hline & & & & & & 4 & 3 & 10824.749 & 0.004 & 10872.458 & 0.003 & 10853.172 & 0.001 \\
\hline & & & & & & 3 & 2 & & & & & 10853.016 & 0.005 \\
\hline \multirow[t]{2}{*}{4} & 1 & 3 & 3 & 1 & 2 & 5 & 4 & 11178.798 & -0.024 & & & & \\
\hline & & & & & & 4 & 3 & 11178.862 & -0.036 & & & & \\
\hline \multirow[t]{3}{*}{4} & 1 & 4 & 3 & 1 & 3 & 5 & 4 & 10649.729 & -0.003 & 10705.692 & 0.004 & 10682.308 & 0.002 \\
\hline & & & & & & 4 & 3 & 10649.825 & -0.005 & & & 10682.403 & 0.002 \\
\hline & & & & & & 3 & 2 & 10649.805 & 0.001 & & & & \\
\hline
\end{tabular}

${ }^{a}$ Observed

${ }^{\mathrm{b}}$ Observed minus calculated 
Table S3. Observed frequencies and residuals $(\mathrm{MHz})$ for the parent species of conformer $2 \mathrm{a}_{2}$ of 4(S)-hydroxyproline.

\begin{tabular}{|c|c|c|c|c|c|c|c|c|c|c|c|c|c|c|c|c|c|c|c|}
\hline \multirow{2}{*}{$\begin{array}{l}\text { J' } \\
2\end{array}$} & \multicolumn{2}{|c|}{$K^{\prime}{ }_{-1} K^{\prime}+1$} & \multirow{2}{*}{$\frac{\text { J" }}{1}$} & \multicolumn{2}{|c|}{$K^{\prime \prime}{ }_{-1} K^{\prime \prime}+1$} & $F^{\prime}$ & $F$ & \multirow{2}{*}{$\begin{array}{c}\text { obs. }^{a} \\
5068.427\end{array}$} & \multirow{2}{*}{$\frac{\text { o.-c. }}{-0.001}$} & \multirow{2}{*}{$\begin{array}{l}J^{\prime} \\
4\end{array}$} & \multicolumn{2}{|c|}{$K_{-1}^{\prime} K_{+1}^{\prime}$} & \multicolumn{3}{|c|}{ J" K" ${ }_{-1} K^{\prime \prime}+1$} & \multicolumn{2}{|c|}{$F^{\prime} F^{\prime \prime}$} & \multirow{2}{*}{$\begin{array}{c}\text { obs. } \\
8800.014\end{array}$} & \multirow{2}{*}{$\begin{array}{l}\text { o.-c. } \\
0.001\end{array}$} \\
\hline & 0 & 2 & & 0 & 1 & 3 & 2 & & & & 0 & 4 & 3 & 1 & 3 & 5 & 4 & & \\
\hline \multirow[t]{2}{*}{2} & 1 & 1 & 1 & 0 & 1 & 3 & 2 & 6644.889 & -0.001 & & & & & & & 4 & 3 & 8801.271 & 0.000 \\
\hline & & & & & & 2 & 1 & 6645.617 & 0.003 & & & & & & & 3 & 2 & 8799.648 & 0.001 \\
\hline \multirow[t]{3}{*}{2} & 1 & 2 & 1 & 0 & 1 & 3 & 2 & 6472.744 & 0.000 & 4 & 1 & 3 & 3 & 0 & 3 & 5 & 4 & 11920.136 & -0.001 \\
\hline & & & & & & 2 & 1 & 6471.188 & 0.003 & & & & & & & 4 & 3 & 11921.293 & 0.000 \\
\hline & & & & & & 1 & 0 & 6474.462 & 0.003 & & & & & & & 3 & 2 & 11919.920 & -0.001 \\
\hline 2 & 1 & 2 & 1 & 1 & 1 & 3 & 2 & 5012.493 & -0.003 & 4 & 1 & 3 & 3 & 1 & 2 & 5 & 4 & 10251.838 & 0.000 \\
\hline 2 & 2 & 0 & 1 & 1 & 0 & 3 & 2 & 9509.197 & -0.002 & & & & & & & 4 & 3 & 10251.960 & 0.000 \\
\hline & & & & & & 2 & 1 & 9507.667 & 0.002 & & & & & & & 3 & 2 & 10251.984 & 0.000 \\
\hline & & & & & & 1 & 0 & 9510.628 & -0.005 & 4 & 1 & 4 & 3 & 0 & 3 & 5 & 4 & 11345.466 & 0.000 \\
\hline 2 & 2 & 0 & 1 & 1 & 1 & 3 & 2 & 9564.635 & 0.002 & & & & & & & 4 & 3 & 11344.204 & 0.000 \\
\hline & & & & & & 2 & 1 & 9566.394 & -0.001 & & & & & & & 3 & 2 & 11345.872 & 0.000 \\
\hline & & & & & & 1 & 0 & 9566.988 & -0.003 & 4 & 1 & 4 & 3 & 1 & 3 & 5 & 4 & 10021.857 & 0.001 \\
\hline 2 & 2 & 1 & 1 & 1 & 0 & 3 & 2 & 9564.736 & 0.000 & & & & & & & 4 & 3 & 10021.924 & -0.002 \\
\hline & & & & & & 2 & $\overline{1}$ & 9565.288 & 0.000 & & & & & & & 3 & 2 & 10021.792 & -0.004 \\
\hline 2 & 2 & 1 & 1 & 1 & 1 & 3 & 2 & 9563.001 & 0.001 & 4 & 2 & 2 & 3 & 1 & 2 & 5 & 4 & 14458.453 & 0.003 \\
\hline & & & & & & 2 & 1 & 9505.961 & -0.002 & & & & & & & 4 & 3 & 14457.142 & 0.000 \\
\hline & & & & & & 1 & 0 & 9507.542 & 0.002 & & & & & & & 3 & 2 & 14458.966 & 0.001 \\
\hline 3 & 0 & 3 & 2 & 0 & 2 & 4 & 3 & 7598.518 & -0.001 & 4 & 2 & 2 & 3 & 1 & 3 & 5 & 4 & 14803.141 & 0.001 \\
\hline & & & & & & 3 & 2 & 7598.480 & -0.004 & & & & & & & 3 & 2 & 14802.821 & -0.005 \\
\hline & & & & & & 2 & 1 & 7598.720 & 0.000 & 4 & 2 & 2 & 3 & 2 & 1 & 5 & 4 & 10155.406 & -0.001 \\
\hline 3 & 1 & 2 & 2 & 0 & 2 & 4 & 3 & 9266.819 & 0.001 & & & & & & & 4 & 3 & 10155.899 & -0.001 \\
\hline & & & & & & 3 & 2 & 9267.818 & 0.001 & & & & & & & 3 & 2 & 10155.289 & -0.001 \\
\hline & & & & & & 2 & 1 & 9266.657 & 0.000 & 4 & 2 & 2 & 3 & 1 & 3 & 4 & 3 & 14804.201 & 0.003 \\
\hline 3 & 1 & 2 & 2 & 1 & 1 & 4 & 3 & 7690.355 & 0.000 & 4 & 2 & 3 & 3 & 1 & 2 & 5 & 4 & 14433.578 & -0.004 \\
\hline & & & & & & 3 & 2 & 7690.649 & -0.001 & & & & & & & 4 & 3 & 14432.064 & 0.003 \\
\hline & & & & & & 2 & 1 & 7690.586 & 0.000 & & & & & & & 3 & 2 & 14434.152 & 0.001 \\
\hline 3 & 1 & 3 & 2 & 0 & 2 & 4 & 3 & 8922.128 & -0.001 & 4 & 2 & 3 & 3 & 1 & 3 & 5 & 4 & 14778.272 & 0.001 \\
\hline & & & & & & 3 & 2 & 8920.760 & -0.002 & & & & & & & 4 & 3 & 14779.116 & -0.002 \\
\hline & & & & & & 2 & 1 & 8922.795 & -0.001 & & & & & & & 3 & 2 & 14778.010 & -0.001 \\
\hline 3 & 1 & 3 & 2 & 1 & 2 & 4 & 3 & 7517.603 & 0.000 & 4 & 2 & 3 & 3 & 2 & 2 & 5 & 4 & 10138.838 & -0.001 \\
\hline & & & & & & 3 & 2 & 7517.812 & 0.000 & & & & & & & 4 & 3 & 10139.238 & 0.001 \\
\hline & & & & & & 2 & 1 & 7518.023 & 0.000 & & & & & & & 3 & 2 & 10138.736 & 0.000 \\
\hline 3 & 2 & 1 & 2 & 1 & 1 & 4 & 3 & 11993.401 & 0.002 & 4 & 3 & 2 & 3 & 3 & 1 & 5 & 4 & 10143.294 & -0.002 \\
\hline & & & & & & 3 & 2 & 11991.893 & 0.001 & 5 & 0 & 5 & 4 & 0 & 4 & 6 & 5 & 12642.261 & -0.001 \\
\hline & & & & & & 2 & 1 & 11994.260 & -0.001 & & & & & & & 5 & 4 & 12642.158 & 0.000 \\
\hline 3 & 2 & 1 & 2 & 1 & 2 & 4 & 3 & 12165.548 & 0.002 & & & & & & & 4 & 3 & 12642.325 & -0.001 \\
\hline & & & & & & 3 & 2 & 12166.323 & 0.002 & 5 & 0 & 5 & 4 & 1 & 4 & 6 & 5 & 11420.419 & 0.001 \\
\hline & & & & & & 2 & 1 & 12165.137 & -0.002 & & & & & & & 5 & 4 & 11421.501 & -0.002 \\
\hline 3 & 2 & 1 & 2 & 2 & 0 & 3 & 2 & 7612.680 & -0.001 & & & & & & & 4 & 3 & 11420.179 & 0.002 \\
\hline 3 & 2 & 2 & 2 & 1 & 1 & 4 & 3 & 11985.100 & 0.002 & 5 & 1 & 4 & 4 & 0 & 4 & 6 & 5 & 14607.945 & -0.001 \\
\hline & & & & & & 3 & 2 & 11983.476 & 0.001 & & & & & & & 5 & 4 & 14609.226 & -0.001 \\
\hline & & & & & & 2 & 1 & 11986.000 & 0.000 & & & & & & & 4 & 3 & 14607.728 & 0.000 \\
\hline 3 & 2 & 2 & 2 & 1 & 2 & 4 & 3 & 12157.244 & -0.001 & 5 & 1 & 4 & 4 & 1 & 3 & 6 & 5 & 12811.432 & 0.001 \\
\hline & & & & & & 3 & 2 & 12157.908 & 0.004 & & & & & & & 5 & 4 & 12811.483 & 0.001 \\
\hline & & & & & & 2 & $\overline{1}$ & 12156.875 & -0.002 & & & & & & & 4 & 3 & 12811.533 & 0.003 \\
\hline
\end{tabular}

\footnotetext{
${ }^{\mathrm{a}}$ Observed
${ }^{\mathrm{O}}$ Observed minus calculated
} 
Table S3 (continued). Observed frequencies and residuals $(\mathrm{MHz})$ for the parent species of conformer $2 \mathrm{a}_{2}$ of $4(S)$-hydroxyproline.

\begin{tabular}{|c|c|c|c|c|c|c|c|c|c|c|c|c|c|c|c|c|c|c|c|}
\hline \multirow[t]{3}{*}{ J' } & \multicolumn{2}{|c|}{$K_{-1}^{\prime} K_{+1}^{\prime}$} & \multirow{3}{*}{$\begin{array}{c}\text { J" } \\
2\end{array}$} & \multicolumn{2}{|c|}{$K^{\prime \prime}{ }_{-1} K^{\prime \prime}$} & \multirow{2}{*}{\multicolumn{2}{|c|}{$\begin{array}{ll}F^{\prime} & F^{\prime \prime} \\
4 & 3\end{array}$}} & \multirow{2}{*}{$\begin{array}{c}\text { obs. }^{a} \\
7605.008\end{array}$} & \multirow{2}{*}{$\begin{array}{l}\text { o.-c. }^{\text {b }} \\
0.004\end{array}$} & \multirow{2}{*}{$\begin{array}{r}\text { J' } \\
5\end{array}$} & \multicolumn{2}{|c|}{$K_{-1}^{\prime} K_{+1}^{\prime}$} & \multicolumn{3}{|c|}{ J" K" ${ }_{-1} K^{\prime \prime}+1$} & \multicolumn{2}{|c|}{$F^{\prime} F^{\prime \prime}$} & \multirow{2}{*}{$\begin{array}{c}\text { obs. } \\
13746.168\end{array}$} & \multirow{2}{*}{$\begin{array}{l}\text { o.-c. } \\
0.003\end{array}$} \\
\hline & 2 & 2 & & 2 & 1 & 4 & & & & & 1 & 5 & 4 & 0 & 4 & 6 & 5 & & \\
\hline & & & & & & 3 & 2 & 7605.970 & 0.004 & & & & & & & 5 & 4 & 13744.993 & -0.003 \\
\hline \multirow[t]{2}{*}{3} & 3 & 0 & 2 & 2 & 0 & 4 & 3 & 15047.731 & 0.003 & & & & & & & 4 & 3 & 13746.447 & 0.000 \\
\hline & & & & & & 3 & 2 & 15047.416 & 0.004 & 5 & 1 & 5 & 4 & 1 & 4 & 6 & 5 & 12524.322 & 0.000 \\
\hline \multirow[t]{3}{*}{3} & 3 & 0 & 2 & 2 & 1 & 4 & 3 & 15049.386 & 0.001 & & & & & & & 5 & 4 & 12524.339 & -0.002 \\
\hline & & & & & & 3 & 2 & 15049.115 & 0.000 & & & & & & & 4 & 3 & 12524.298 & 0.000 \\
\hline & & & & & & 2 & $\overline{1}$ & 15049.277 & -0.005 & 6 & 0 & 6 & 5 & 0 & 5 & 7 & 6 & 15153.112 & -0.001 \\
\hline \multirow[t]{3}{*}{3} & 3 & 1 & 2 & 2 & 0 & 4 & 3 & 15047.698 & 0.001 & & & & & & & 6 & 5 & 15153.240 & -0.001 \\
\hline & & & & & & 3 & 2 & 15047.382 & 0.000 & & & & & & & 5 & 4 & 15153.284 & -0.003 \\
\hline & & & & & & 2 & 1 & 15047.620 & 0.001 & 6 & 0 & 6 & 5 & 1 & 5 & 7 & 6 & 14049.165 & 0.000 \\
\hline \multirow[t]{3}{*}{3} & 3 & 1 & 2 & 2 & 1 & 4 & 3 & 15049.355 & -0.001 & & & & & & & 6 & 5 & 14049.337 & 0.000 \\
\hline & & & & & & 3 & 2 & 15049.083 & -0.002 & & & & & & & 5 & 4 & 14050.276 & 0.001 \\
\hline & & & & & & 2 & 1 & 15049.250 & -0.002 & 6 & 1 & 5 & 5 & 1 & 4 & 7 & 6 & 15368.572 & 0.001 \\
\hline \multirow[t]{4}{*}{4} & 0 & 4 & 3 & 0 & 3 & 5 & 4 & 10123.623 & 0.001 & & & & & & & 6 & 5 & 15368.583 & -0.001 \\
\hline & & & & & & 4 & 3 & 10123.552 & 0.003 & & & & & & & 5 & 4 & 15368.642 & 0.000 \\
\hline & & & & & & 3 & 2 & 10123.724 & 0.001 & 6 & 1 & 6 & 5 & 1 & 5 & 7 & 6 & 15024.937 & 0.003 \\
\hline & & & & & & & & & & & & & & & & 6 & 5 & 15024.929 & 0.001 \\
\hline
\end{tabular}

\footnotetext{
abserved

${ }^{\mathrm{b}}$ Observed minus calculated
} 
Table S4. Observed frequencies and residuals $(\mathrm{MHz})$ for the parent species of conformer $1 \mathrm{~b}_{1}$ of 4(R)-hydroxyproline.

\begin{tabular}{|c|c|c|c|c|c|c|c|c|c|c|c|c|c|c|c|c|c|c|c|}
\hline \multirow{2}{*}{$\begin{array}{l}J^{\prime} \\
3\end{array}$} & \multicolumn{2}{|c|}{$\mathrm{K}_{-1}^{\prime} \mathrm{K}^{\prime}+1$} & \multirow{2}{*}{$\frac{\mathrm{J} "}{2}$} & \multicolumn{2}{|c|}{$K^{\prime \prime}{ }_{-1} K^{\prime \prime}+1$} & \multirow[t]{2}{*}{$F^{\prime}$} & \multirow{2}{*}{$\frac{F^{\prime \prime}}{3}$} & \multirow{2}{*}{$\begin{array}{c}\text { obs. }^{a} \\
6472.396\end{array}$} & \multirow{2}{*}{$\frac{0 .-c .^{b}}{0.002}$} & \multirow{2}{*}{$\begin{array}{l}J^{\prime} \\
6\end{array}$} & \multicolumn{2}{|c|}{$K^{\prime}-1 K^{\prime}+1$} & \multicolumn{3}{|c|}{ J" K"-1 K"'+1 } & \multicolumn{2}{|c|}{$F^{\prime} F^{\prime \prime}$} & \multirow{2}{*}{$\begin{array}{c}\text { obs. } \\
12424.670\end{array}$} & \multirow{2}{*}{$\begin{array}{r}\text { o.-c. } \\
-0.003\end{array}$} \\
\hline & 1 & 2 & & 1 & 1 & & & & & & 2 & 5 & 5 & 2 & 4 & 7 & 6 & & \\
\hline & & & & & & 3 & 2 & 6472.298 & 0.000 & & & & & & & 5 & 4 & 12424.670 & -0.005 \\
\hline & & & & & & 2 & 1 & 6472.465 & -0.003 & 6 & 2 & 5 & 6 & 1 & 6 & 7 & 7 & 9169.367 & 0.000 \\
\hline \multirow[t]{3}{*}{4} & 0 & 4 & 3 & 0 & 3 & 5 & 4 & 8226.709 & -0.002 & 6 & 3 & 3 & 5 & 3 & 2 & 7 & 6 & 12514.285 & 0.000 \\
\hline & & & & & & 4 & 3 & 8226.652 & 0.001 & & & & & & & 6 & 5 & 12514.200 & 0.002 \\
\hline & & & & & & 3 & 2 & 8226.685 & 0.000 & & & & & & & 5 & 4 & 12514.304 & 0.004 \\
\hline 4 & 1 & 3 & 3 & 1 & 2 & 5 & 4 & 8619.608 & -0.002 & 7 & 0 & 7 & 6 & 0 & 6 & 8 & 7 & 14132.673 & 0.000 \\
\hline & & & & & & 4 & 3 & 8619.562 & 0.002 & & & & & & & 7 & 6 & 14132.601 & 0.000 \\
\hline & & & & & & 3 & 2 & 8619.632 & -0.001 & 7 & 1 & 6 & 6 & 1 & 5 & 8 & 7 & 14989.777 & -0.001 \\
\hline 4 & 1 & 4 & 3 & 0 & 3 & 5 & 4 & 9969.074 & 0.003 & & & & & & & 7 & 6 & 14989.740 & 0.000 \\
\hline 4 & 1 & 4 & 3 & 1 & 3 & 5 & 4 & 7963.060 & 0.001 & & & & & & & 6 & 5 & 14989.777 & -0.006 \\
\hline & & & & & & 3 & 2 & 7963.018 & 0.005 & 7 & 1 & 7 & 6 & 1 & 6 & 8 & 7 & 13869.908 & 0.003 \\
\hline 5 & 0 & 5 & 4 & 0 & 4 & 6 & 5 & 10225.987 & 0.002 & & & & & & & 7 & 6 & 13869.869 & -0.004 \\
\hline & & & & & & 5 & 4 & 10225.917 & 0.000 & & & & & & & 6 & 5 & 13869.890 & 0.001 \\
\hline & & & & & & 4 & 3 & 10225.968 & -0.004 & 7 & 2 & 5 & 6 & 2 & 4 & 8 & 7 & 14879.925 & -0.004 \\
\hline & & & & & & 5 & 5 & 10225.412 & 0.003 & & & & & & & 7 & 6 & 14879.954 & 0.004 \\
\hline & & & & & & 4 & 4 & 10226.612 & 0.001 & & & & & & & 6 & 5 & 14879.925 & 0.001 \\
\hline 5 & 1 & 4 & 4 & 1 & 3 & 6 & 5 & 10757.265 & -0.004 & 7 & 2 & 6 & 6 & 2 & 5 & 8 & 7 & 14474.800 & 0.000 \\
\hline & & & & & & 5 & 4 & 10757.233 & 0.002 & 7 & 3 & 4 & 6 & 3 & 3 & 8 & 7 & 14623.492 & 0.003 \\
\hline & & & & & & 4 & 3 & 10757.277 & -0.001 & & & & & & & 6 & 5 & 14623.492 & -0.002 \\
\hline 5 & 1 & 5 & 4 & 1 & 4 & 6 & 5 & 9940.030 & 0.000 & 7 & 3 & 5 & 6 & 3 & 4 & 8 & 7 & 14591.693 & 0.001 \\
\hline & & & & & & 5 & 4 & 9939.979 & -0.005 & & & & & & & 7 & 6 & 14591.636 & 0.004 \\
\hline & & & & & & 5 & 5 & 9939.227 & -0.002 & & & & & & & 6 & 5 & 14591.693 & -0.004 \\
\hline & & & & & & 4 & 4 & 9940.946 & -0.004 & 8 & 0 & 8 & 7 & 0 & 7 & 9 & 8 & 16049.207 & -0.001 \\
\hline 5 & 2 & 3 & 4 & 2 & 2 & 6 & 5 & 10525.178 & 0.003 & & & & & & & 8 & 7 & 16049.146 & 0.005 \\
\hline & & & & & & 5 & 4 & 10525.147 & 0.002 & & & & & & & 7 & 6 & 16049.207 & 0.004 \\
\hline & & & & & & 4 & 3 & 10525.178 & -0.001 & 8 & 1 & 7 & 7 & 1 & 6 & 9 & 8 & 17076.070 & -0.001 \\
\hline 5 & 2 & 4 & 4 & 2 & 3 & 6 & 5 & 10366.452 & -0.002 & & & & & & & 8 & 7 & 17076.027 & 0.000 \\
\hline & & & & & & 5 & 4 & 10366.362 & -0.001 & & & & & & & 7 & 6 & 17076.070 & -0.005 \\
\hline & & & & & & 4 & 3 & 10366.465 & 0.001 & 8 & 1 & 8 & 7 & 1 & 7 & 9 & 8 & 15822.466 & 0.002 \\
\hline 5 & 3 & 2 & 4 & 3 & 1 & 6 & 5 & 10415.802 & 0.000 & & & & & & & 8 & 7 & 15822.431 & -0.004 \\
\hline & & & & & & 5 & 4 & 10415.636 & -0.003 & & & & & & & 7 & 6 & 15822.449 & -0.003 \\
\hline & & & & & & 4 & 3 & 10415.841 & -0.003 & 8 & 2 & 6 & 7 & 2 & 5 & 9 & 8 & 17079.750 & -0.004 \\
\hline 6 & 0 & 6 & 5 & 0 & 5 & 7 & 6 & 12193.804 & 0.000 & & & & & & & 8 & 7 & 17079.781 & 0.004 \\
\hline & & & & & & 6 & 5 & 12193.731 & -0.001 & & & & & & & 7 & 6 & 17079.750 & 0.000 \\
\hline & & & & & & 6 & 6 & 12193.153 & -0.003 & 8 & 2 & 7 & 7 & 2 & 6 & 9 & 8 & 16515.647 & -0.001 \\
\hline & & & & & & 5 & 5 & 12194.491 & 0.002 & & & & & & & 8 & 7 & 16515.609 & 0.000 \\
\hline 6 & 0 & 6 & 5 & 1 & 5 & 7 & 6 & 10737.398 & -0.002 & & & & & & & 7 & 6 & 16515.647 & 0.001 \\
\hline & & & & & & 6 & 5 & 10737.557 & 0.004 & 8 & 3 & 5 & 7 & 3 & 4 & 9 & 8 & 16746.935 & 0.003 \\
\hline & & & & & & 5 & 4 & 10737.346 & 0.001 & & & & & & & 8 & 7 & 16746.908 & -0.004 \\
\hline 6 & 1 & 5 & 5 & 1 & 4 & 7 & 6 & 12881.987 & 0.000 & & & & & & & 7 & 6 & 16746.935 & 0.003 \\
\hline & & & & & & 6 & 5 & 12881.954 & 0.003 & 8 & 3 & 6 & 7 & 3 & 5 & 9 & 8 & 16684.373 & 0.000 \\
\hline 6 & 1 & 6 & 5 & 1 & 5 & 7 & 6 & 11909.115 & 0.001 & & & & & & & 8 & 7 & 16684.332 & -0.001 \\
\hline & & & & & & 6 & 5 & 11909.077 & 0.000 & & & & & & & 7 & 6 & 16684.373 & -0.002 \\
\hline & & & & & & 5 & 4 & 11909.095 & 0.003 & 9 & 0 & 9 & 8 & 0 & 8 & 10 & 9 & 17951.873 & -0.003 \\
\hline 6 & 2 & 4 & 5 & 2 & 3 & 7 & 6 & 12692.314 & -0.001 & & & & & & & 9 & 8 & 17951.818 & 0.001 \\
\hline & & & & & & 5 & 4 & 12692.314 & 0.003 & & & & & & & 8 & 7 & 17951.873 & 0.001 \\
\hline
\end{tabular}

\footnotetext{
${ }^{a}$ Observed

${ }^{\mathrm{b}}$ Observed minus calculated
} 
Table S5. Observed frequencies and residuals (MHz) for the ${ }^{13} \mathrm{C}_{\alpha}$ isotopomer of conformer $1 \mathrm{~b}_{1}$ of 4(R)-hydroxyproline.

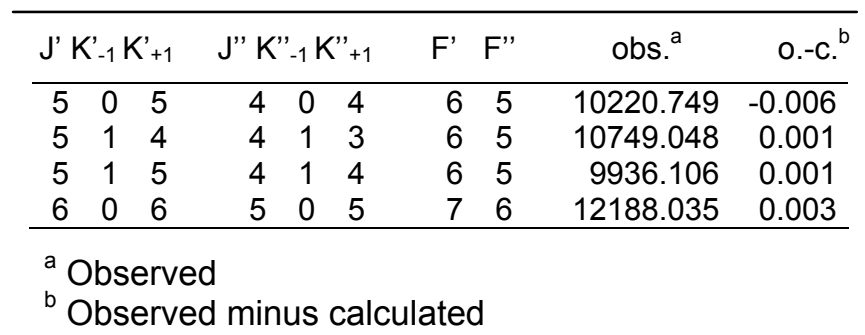


Table S6. Observed frequencies and residuals $(\mathrm{MHz})$ for the monodeuterated isotopomers of conformer $1 \mathrm{~b}_{1}$ of 4(R)-hydroxyproline.

\begin{tabular}{|c|c|c|c|c|c|c|c|c|c|c|c|}
\hline \multirow{2}{*}{\multicolumn{3}{|c|}{$J^{\prime} K_{-1}^{\prime} K_{+1}^{\prime}$}} & \multirow{2}{*}{\multicolumn{3}{|c|}{ J" $K^{\prime \prime}{ }_{-1} K^{\prime \prime}+1$}} & \multirow[b]{2}{*}{$F^{\prime}$} & \multirow[b]{2}{*}{ F" } & \multicolumn{2}{|c|}{ ND } & \multicolumn{2}{|c|}{ COOD } \\
\hline & & & & & & & & obs. ${ }^{a}$ & o.-c. ${ }^{b}$ & obs. & o.-c. \\
\hline \multirow[t]{3}{*}{5} & 0 & 5 & 4 & 0 & 4 & 6 & 5 & 10173.186 & -0.002 & 10142.930 & 0.002 \\
\hline & & & & & & 5 & 4 & 10173.119 & 0.000 & 10142.859 & 0.000 \\
\hline & & & & & & 4 & 3 & & & 10142.908 & -0.008 \\
\hline \multirow[t]{3}{*}{5} & 1 & 4 & 4 & 1 & 3 & 6 & 5 & 10703.287 & 0.002 & 10689.552 & -0.002 \\
\hline & & & & & & 5 & 4 & 10703.250 & 0.003 & 10689.512 & -0.003 \\
\hline & & & & & & 4 & 3 & 10703.302 & 0.006 & 10689.564 & 0.000 \\
\hline \multirow[t]{3}{*}{5} & 1 & 5 & 4 & 1 & 4 & 6 & 5 & 9892.277 & -0.002 & 9856.503 & 0.000 \\
\hline & & & & & & 5 & 4 & 9892.229 & -0.004 & 9856.452 & -0.004 \\
\hline & & & & & & 4 & 3 & 9892.251 & 0.003 & 9856.474 & 0.003 \\
\hline \multirow[t]{3}{*}{6} & 0 & 6 & 5 & 0 & 5 & 7 & 6 & 12129.643 & 0.002 & 12090.183 & -0.007 \\
\hline & & & & & & 6 & 5 & 12129.569 & 0.002 & 12090.125 & 0.009 \\
\hline & & & & & & 5 & 4 & 12129.631 & -0.002 & & \\
\hline \multirow[t]{3}{*}{6} & 1 & 5 & 5 & 1 & 4 & 7 & 6 & 12816.617 & 0.001 & 12798.802 & 0.001 \\
\hline & & & & & & 6 & 5 & 12816.572 & -0.007 & 12798.773 & 0.008 \\
\hline & & & & & & 5 & 4 & 12816.617 & -0.005 & 12798.802 & -0.006 \\
\hline \multirow[t]{3}{*}{6} & 1 & 6 & 5 & 1 & 5 & 7 & 6 & 11851.562 & 0.000 & 11807.819 & 0.001 \\
\hline & & & & & & 6 & 5 & 11851.522 & -0.002 & 11807.779 & -0.002 \\
\hline & & & & & & 5 & 4 & 11851.543 & 0.003 & 11807.800 & 0.003 \\
\hline
\end{tabular}

${ }^{\mathrm{a}}$ Observed 
Table S7. Observed frequencies and residuals $(\mathrm{MHz})$ for the parent species of conformer $2 \mathrm{~b}_{2}$ of 4(R)-hydroxyproline.

\begin{tabular}{|c|c|c|c|c|c|c|c|c|c|c|c|c|c|c|c|c|c|c|c|}
\hline \multirow{2}{*}{$\begin{array}{l}\text { J' } \\
4\end{array}$} & \multicolumn{2}{|c|}{$K_{-1}^{\prime} K^{\prime}+1$} & \multirow{2}{*}{$\frac{J^{\prime \prime}}{3}$} & \multicolumn{2}{|c|}{$K^{\prime \prime} K^{\prime \prime}+1$} & \multirow[t]{2}{*}{$F^{\prime}$} & \multirow{2}{*}{$\frac{F^{\prime \prime}}{4}$} & \multirow{2}{*}{$\begin{array}{c}\text { obs. }^{a} \\
8196.861\end{array}$} & \multirow{2}{*}{$\begin{array}{c}\text { o.-c. } \\
-0.001\end{array}$} & \multirow{2}{*}{$\begin{array}{c}\text { J' } \\
6\end{array}$} & \multicolumn{2}{|c|}{$\mathrm{K}_{-1}^{\prime} \mathrm{K}_{+1}^{\prime}$} & \multicolumn{3}{|c|}{ J" K" ${ }_{-1} K^{\prime \prime}+1$} & \multicolumn{2}{|c|}{$F^{\prime} F^{\prime \prime}$} & \multirow{2}{*}{$\begin{array}{c}\text { obs. } \\
12415.544\end{array}$} & \multirow{2}{*}{$\begin{array}{c}\text { o.-C. } \\
-0.001\end{array}$} \\
\hline & 0 & 4 & & 0 & 3 & & & & & & 4 & 3 & 5 & 4 & 2 & 6 & 5 & & \\
\hline & & & & & & 4 & 3 & 8196.941 & 0.001 & 6 & 5 & 1 & 5 & 5 & 0 & 7 & 6 & 12409.760 & 0.002 \\
\hline \multirow[t]{3}{*}{4} & 1 & 3 & 3 & 1 & 2 & 5 & 4 & 8546.363 & 0.000 & & & & & & & 6 & 5 & 12409.578 & 0.000 \\
\hline & & & & & & 4 & 3 & 8546.338 & -0.004 & & & & & & & 5 & 4 & 12409.793 & -0.006 \\
\hline & & & & & & 3 & 2 & 8546.293 & 0.003 & 6 & 5 & 2 & 5 & 5 & 1 & 7 & 6 & 12409.760 & 0.003 \\
\hline 4 & 1 & 4 & 3 & 1 & 3 & 5 & 4 & 7951.587 & 0.000 & & & & & & & 6 & 5 & 12409.578 & 0.000 \\
\hline 4 & 2 & 2 & 3 & 2 & 1 & 5 & 4 & 8322.427 & 0.002 & & & & & & & 5 & 4 & 12409.793 & -0.005 \\
\hline \multirow[t]{3}{*}{5} & 0 & 5 & 4 & 0 & 4 & 6 & 5 & 10199.288 & 0.003 & 7 & 0 & 7 & 6 & 0 & 6 & 8 & 7 & 14124.836 & 0.003 \\
\hline & & & & & & 5 & 4 & 10199.386 & 0.001 & & & & & & & 7 & 6 & 14124.946 & 0.000 \\
\hline & & & & & & 4 & 3 & 10199.267 & -0.002 & & & & & & & 6 & 5 & 14124.817 & -0.003 \\
\hline \multirow[t]{3}{*}{5} & 1 & 4 & 4 & 1 & 3 & 6 & 5 & 10669.263 & 0.002 & 7 & 1 & 6 & 6 & 1 & 5 & 8 & 7 & 14881.571 & 0.001 \\
\hline & & & & & & 5 & 4 & 10669.263 & -0.002 & & & & & & & 7 & 6 & 14881.605 & 0.000 \\
\hline & & & & & & 4 & 3 & 10669.213 & -0.001 & & & & & & & 6 & 5 & 14881.545 & 0.002 \\
\hline \multirow[t]{2}{*}{5} & 1 & 5 & 4 & 1 & 4 & 6 & 5 & 9928.278 & 0.002 & 7 & 1 & 7 & 6 & 1 & 6 & 8 & 7 & 13861.586 & 0.000 \\
\hline & & & & & & 5 & 4 & 9928.295 & -0.001 & & & & & & & 7 & 6 & 13861.621 & 0.005 \\
\hline \multirow[t]{3}{*}{5} & 2 & 3 & 4 & 2 & 2 & 6 & 5 & 10441.484 & -0.004 & & & & & & & 6 & 5 & 13861.600 & 0.004 \\
\hline & & & & & & 5 & 4 & 10441.354 & 0.000 & 7 & 2 & 5 & 6 & 2 & 4 & 8 & 7 & 14740.985 & -0.001 \\
\hline & & & & & & 4 & 3 & 10441.504 & 0.004 & & & & & & & 7 & 6 & 14740.874 & -0.004 \\
\hline 5 & 2 & 4 & 4 & 2 & 3 & 6 & 5 & 10313.061 & 0.002 & & & & & & & 6 & 5 & 14740.985 & -0.004 \\
\hline & & & & & & 5 & 4 & 10313.031 & 0.002 & 7 & 2 & 6 & 6 & 2 & 5 & 8 & 7 & 14407.531 & -0.001 \\
\hline & & & & & & 4 & 3 & 10313.061 & 0.002 & & & & & & & 6 & 5 & 14407.531 & 0.003 \\
\hline 5 & 3 & 2 & 4 & 3 & 1 & 6 & 5 & 10352.442 & 0.001 & 7 & 3 & 4 & 6 & 3 & 3 & 8 & 7 & 14525.493 & 0.005 \\
\hline & & & & & & 5 & 4 & 10352.313 & -0.001 & & & & & & & 7 & 6 & 14525.419 & 0.001 \\
\hline & & & & & & 4 & 3 & 10352.469 & 0.000 & 7 & 3 & 5 & 6 & 3 & 4 & 8 & 7 & 14502.749 & 0.000 \\
\hline 5 & 3 & 3 & 4 & 3 & 2 & 6 & 5 & 10348.596 & -0.004 & & & & & & & 7 & 6 & 14502.697 & 0.000 \\
\hline 5 & 4 & 1 & 4 & 4 & 0 & 6 & 5 & 10342.460 & -0.002 & & & & & & & 6 & 5 & 14502.749 & -0.003 \\
\hline & & & & & & 5 & 4 & 10342.260 & -0.004 & 7 & 4 & 3 & 6 & 4 & 2 & 8 & 7 & 14491.949 & -0.001 \\
\hline & & & & & & 4 & 3 & 10342.523 & 0.003 & & & & & & & 7 & 6 & 14491.871 & 0.003 \\
\hline 5 & 4 & 2 & 4 & 4 & 1 & 5 & 4 & 10342.233 & 0.003 & 7 & 4 & 4 & 6 & 4 & 3 & 8 & 7 & 14491.444 & -0.001 \\
\hline & & & & & & 4 & 3 & 10342.490 & 0.004 & & & & & & & 7 & 6 & 14491.367 & 0.004 \\
\hline 6 & 0 & 6 & 5 & 0 & 5 & 7 & 6 & 12175.013 & 0.000 & & & & & & & 6 & 5 & 14491.458 & 0.001 \\
\hline & & & & & & 6 & 5 & 12175.116 & -0.002 & 8 & 0 & 8 & 7 & 0 & 7 & 9 & 8 & 16052.984 & 0.003 \\
\hline & & & & & & 5 & 4 & 12174.986 & -0.004 & & & & & & & 8 & 7 & 16053.089 & -0.001 \\
\hline 6 & 1 & 5 & 5 & 1 & 4 & 7 & 6 & 12782.009 & -0.002 & & & & & & & 7 & 6 & 16052.971 & -0.001 \\
\hline & & & & & & 6 & 5 & 12782.034 & 0.002 & 8 & 1 & 7 & 7 & 1 & 6 & 9 & 8 & 16964.429 & 0.001 \\
\hline & & & & & & 5 & 4 & 12781.981 & 0.003 & & & & & & & 8 & 7 & 16964.476 & -0.002 \\
\hline 6 & 1 & 6 & 5 & 1 & 5 & 7 & 6 & 11898.420 & -0.006 & & & & & & & 7 & 6 & 16964.408 & 0.001 \\
\hline & & & & & & 6 & 5 & 11898.453 & 0.002 & 8 & 1 & 8 & 7 & 1 & 7 & 9 & 8 & 15817.668 & -0.002 \\
\hline & & & & & & 5 & 4 & 11898.439 & -0.001 & & & & & & & 8 & 7 & 15817.701 & 0.000 \\
\hline
\end{tabular}


Table $\mathbf{S 7}$ (continued). Observed frequencies and residuals $(\mathrm{MHz})$ for the parent species of conformer $2 \mathrm{~b}_{2}$ of $4(R)$-hydroxyproline.

\begin{tabular}{|c|c|c|c|c|c|c|c|c|c|c|c|c|c|c|c|c|c|c|c|}
\hline \multirow{2}{*}{$\begin{array}{l}J^{\prime} \\
6\end{array}$} & \multicolumn{2}{|c|}{$\mathrm{K}_{-1}^{\prime} \mathrm{K}_{+1}^{\prime}$} & \multirow{2}{*}{$\begin{array}{l}\mathrm{J} " \\
5\end{array}$} & \multicolumn{2}{|c|}{$K^{\prime \prime} K^{\prime \prime}+1$} & \multirow[t]{2}{*}{$\mathrm{F}$} & \multirow{2}{*}{$\begin{array}{l}F^{\prime \prime} \\
6\end{array}$} & \multirow{2}{*}{$\begin{array}{c}\text { obs. }^{a} \\
12581.665\end{array}$} & \multirow{2}{*}{$\begin{array}{c}\text { o.-c. } \\
-0.004\end{array}$} & \multirow{2}{*}{$\begin{array}{l}\text { J' } \\
8\end{array}$} & \multicolumn{2}{|c|}{$\mathrm{K}_{-1}^{\prime} \mathrm{K}_{+1}^{\prime}$} & \multicolumn{3}{|c|}{ J" K" ${ }_{-1}^{\prime} K^{\prime \prime}+1$} & \multicolumn{2}{|c|}{$F^{\prime} F^{\prime \prime}$} & \multirow{2}{*}{$\begin{array}{c}\text { obs. } \\
16913.926\end{array}$} & \multirow{2}{*}{$\begin{array}{r}\text { o.-C. } \\
-0.001\end{array}$} \\
\hline & 2 & 4 & & 2 & 3 & & & & & & 2 & 6 & 7 & 2 & 5 & 9 & 8 & & \\
\hline & & & & & & 6 & 5 & 12581.551 & 0.002 & & & & & & & 7 & 6 & 16913.926 & -0.001 \\
\hline & & & & & & 5 & 4 & 12581.679 & 0.004 & 8 & 2 & 7 & 7 & 2 & 6 & 9 & 8 & 16443.996 & -0.004 \\
\hline \multirow[t]{2}{*}{6} & 2 & 5 & 5 & 2 & 4 & 7 & 6 & 12363.555 & -0.002 & & & & & & & 8 & 7 & 16444.012 & -0.004 \\
\hline & & & & & & 5 & 4 & 12363.555 & 0.000 & & & & & & & 7 & 6 & 16443.996 & 0.000 \\
\hline \multirow[t]{3}{*}{6} & 3 & 3 & 5 & 3 & 2 & 7 & 6 & 12434.856 & -0.005 & 8 & 3 & 5 & 7 & 3 & 4 & 9 & 8 & 16626.990 & 0.001 \\
\hline & & & & & & 6 & 5 & 12434.770 & -0.004 & & & & & & & 8 & 7 & 16626.928 & 0.004 \\
\hline & & & & & & 5 & 4 & 12434.876 & 0.003 & & & & & & & 7 & 6 & 16626.990 & -0.003 \\
\hline \multirow[t]{2}{*}{6} & 4 & 2 & 5 & 4 & 1 & 7 & 6 & 12415.817 & -0.001 & 8 & 3 & 6 & 7 & 3 & 5 & 9 & 8 & 16582.090 & 0.001 \\
\hline & & & & & & 6 & 5 & 12415.698 & 0.001 & & & & & & & 7 & 6 & 16582.090 & 0.000 \\
\hline
\end{tabular}

${ }^{a}$ Observed

${ }^{\mathrm{b}}$ Observed minus calculated 